\title{
New Method for Monitoring the Process of Freeze Drying of Biological Materials
}

\author{
Nikolay Alkeev, ${ }^{1,2}$ Stanislav Averin, ${ }^{1}$ and Svetlana von Gratowski ${ }^{1}$
}

Received 28 November 2014; accepted 18 May 2015; published online 29 May 2015

\begin{abstract}
A capacitive sensor was proposed and tested for the monitoring and control of a freeze drying process of a vaccine against the Newcastle disease of birds. The residual moisture of the vaccine was measured by the thermogravimetric method. The vaccine activity was determined by titration in chicken embryos. It was shown that, at the stages of freezing and primary drying, a capacitive sensor measured the fraction of unfrozen liquid phase in a material and allowed one to control the sublimation stage of drying in an optimal way. This prevented the foaming of the material and shortened the total drying time approximately twice. The control range at the sublimation stage of drying expanded up to $-70^{\circ} \mathrm{C}$. It was found at the final stage of drying that the signal of a capacitive sensor passed through a maximum value. We supposed that this maximum corresponds to the minimum of intramolecular mobility of biological macromolecules and hence to the optimal residual moisture of the material, which ensures long-term preservation of its activity. We also suppose that using the capacitive sensor at the final stage of drying allows one to more precisely detect the time when the residual moisture of dried material reaches the optimal value.
\end{abstract}

KEY WORDS: biological materials; capacitive sensor; freeze drying; optimal residual moisture.

At present, most biological materials containing live viruses or bacteria are exposed to lyophilization (i.e., drying from the frozen state); this ensures long-term preservation of their activity. Typically, this process consists of preliminary freezing and subsequent freeze drying. The latter process, in turn, consists of two stages: primary drying and secondary drying. During primary drying or sublimation, frozen water is removed from a biological product under vacuum and at temperatures below $0^{\circ} \mathrm{C}$. At this stage, the drying rate is limited because of the foaming of a product that occurs due to its high temperature and the excess amount of liquid phase in it. The secondary drying, or final stage, begins after the end of the sublimation stage and occurs at temperatures above $0^{\circ} \mathrm{C}$. The goal of the secondary drying is to bring the residual moisture of a biological product to an optimum level, which provides longterm preservation of its activity. Note that the moisture content both above and below the optimum value reduces the effective life of biological materials $(1,2)$

To increase the shelf life of biological products, the following should be investigated: (1) the influence of the composition of the dried biological product and the residual moisture on the change in its activity over the time (3); (2) it is needed to optimize the sublimation drying process for different types of biological products (4). For the investigation of the of the state of water in the dried biologic drugs and the influence of the humidity of the biological on the change in their activity during shelf life, different physical

\footnotetext{
${ }^{1}$ Kotel'nikov Institute of Radio Engineering and Electronics (Fryazino Branch), Russian Academy of Sciences, Vvedenski sq. 1, Fryazino, Moscow Region, 141190, Russia.

${ }^{2}$ To whom correspondence should be addressed. (e-mail: nikolayalkeev@mail.ru)
}

methods are used such as neutron scattering (5), nuclear magnetic resonance (NMR) (6,7), Raman spectroscopy (8), infrared spectroscopy, differential scanning calorimetry, thermal activity monitor (9), and gravimetric sorption analysis (10). The investigations using these methods allow to find an optimum composition of a protective medium for biologics and to determine its optimal residual moisture.

At all stages of the freeze drying, the parameters of the material and the parameters of the drying process (temperature of a material, the shelf temperature, the condenser temperature, the pressure in the sublimation chamber, etc.) are also monitored. According to these data, the mode of the process is selected to conduct him for the minimum time and get the best product quality (11). Usually during the drying process, the temperature is measured in several vials with biologic located on different shelves. The sharp increase of the temperature indicates the end of primary drying and the beginning of the secondary drying. The finish of the sublimation stage is revealed by a sharp decrease of the partial pressure of water vapor in the sublimation chamber $(12,13)$. Note that the partial pressure of water vapor in the sublimation chamber does not characterize the state of the biological product to be dried and it is an indirect parameter. For monitoring and controlling the process of freeze drying, it is important to use the own properties of biological materials. In (14), a resistivity sensor placed in a frozen biological material was proposed to control the primary stage of freeze drying. A disadvantage of this method is that one cannot establish an unambiguous relationship between the amount of liquid phase in the frozen material and the value of resistivity: the resistance of the sensor depends not only on the amount of liquid phase but also on the concentration of dissolved salts. Another disadvantage of the resistivity sensor is that, when the temperature 
decreases, the resistivity of the material sharply increases to values that are difficult to measure, which makes impossible the control of the sublimation stage with this sensor.

In $(15,16)$, the interesting methods for determining the moisture of biological materials during secondary drying were proposed. These methods are based on the measurement of the partial pressure of water vapors in the sublimation chamber by NIR spectroscopy or Raman spectroscopy. Note that this method is indirect and requires laborious calibration to establish a correspondence between the current moisture of the biological material in vials and the pressure of water vapor in the sublimation chamber.

It should be noted that one has to carry out a series of long-term experiments to find the optimal residual moisture of a biological product. These experiments result in the lifetimes of biological samples with various residual moistures. As the optimal residual moisture of a biological product, one takes the value that provides the longest term preservation of its activity.

However, finding the optimal conditions of freeze drying has traditionally been a process of trial and error and required several experimental runs (17). Note also that the freeze drying process is time-consuming and labor intensive.

A promising method for the investigation of the properties of biological materials is dielcometry $(18,19)$. This method is relatively simple and very informative since it gives information about the structure of biological macromolecules and the state and role of water in the biological material, etc. This method was used in (2022) for monitoring biological materials at the primary stage of freeze drying. In (20), authors had found an anomalous lowfrequency dispersion of the dielectric permittivity in the biological under study and explain this phenomenon by the proton transfer among water molecules, connected by hydrogen bonds The dielectric relaxation time turned out to be sensitive to the loss of moisture content in the product, and the authors suggested to use of this phenomenon to determine the end point of the freeze drying process. The authors mounted the electrodes of the capacitive sensor on the outer surface of vials with the material to be dried. This approach allows monitoring the sublimation rate and determining the end of the primary stage of freeze drying. Unfortunately, the sensitivity of the capacitive sensor of this design is not enough for the reliable monitoring of the stage of secondary drying.

In this paper, a new design of a capacitive sensor and measurement technique are proposed that enable monitoring all stages of the drying process: the freezing stage, the sublimation stage, and the final stage. During freezing and the sublimation stages, the sensor monitors the amount of liquid phase in the frozen material. This allows an optimal control during the whole sublimation stage which prevents the foaming of the material and significantly reduces the total drying time. The sensor also fixes the end of the sublimation stage and the beginning of the final stage of drying. At this stage, the high sensitivity of the measuring system enables one to discover that there is a certain time interval when the signal of the capacitive sensor passes through a maximum. We believe that this maximum corresponds to the minimum of the molecular mobility of biological macromolecules and the optimal residual moisture of the material to be dried.

\section{EXPERIMENTAL TECHNIQUES AND DATA PROCESSING}

As a test, biological material at the freezing stage, skimmed milk (produced by federal state unitary enterprise
"Schelkovo biokombinat"), and a protective medium based on peptone (produced in Semipalatinsk) were used. As a test material for the freeze drying process, a vaccine against Newcastle disease strain of Boron-74 VGNKI with skimmed milk as a protective medium was used. The vaccine, $6 \mathrm{ml}$, was packed in 20-ml vials. A miniature resistance thermometer was placed in the capacitive sensor to measure the temperature of the dried biological material.

Before drying, the trays with the vials were placed in the refrigerator, where the biological materials were frozen to about $-40^{\circ} \mathrm{C}$. The drying of the material was carried out in a TG-50 freeze dryer.

A distinctive feature of proposed approach is that the electrodes of the capacitive sensor were connected to the selfoscillating circuit and the frequency of this self-oscillator was measured. This approach is widely used in science and technology (23) and allows to significantly improve the measurement accuracy of the sensor frequency and hence the measurement accuracy of the dielectric permittivity of a biological material. In the self-oscillating method, the measurement of frequency consists in counting the number of pulses in a given time interval. The freeze drying usually takes from 1 to 3 days, and the counting time interval should be set to a large value. In our measurements, the counting time for the freezing and sublimation stages was $20 \mathrm{~s}$ and for the final drying stage was $200 \mathrm{~s}$. The accuracy of proposed method is higher compared with that in (20), because the electrodes of the capacitive sensor are placed directly in the material rather than outside a vial.

Figure 1 shows the measurement setup. The capacitive sensor 1 was similar to the vials for the material to be dried but had a greater diameter. The sensor was placed on a tray together with ordinary vials and was filled with a liquid biological material to the same level as in the vials. The sensor electrodes were arranged horizontally, and the state of the material from the bottom of the sensor to the level of about 0.2 of the height of the biological product in the vials was monitored. The electrodes were made of stainless steel and had a number of holes that provided an outlet for water vapor. The sensor was covered by a Teflon stopper in which the selfoscillator was mounted with electrodes connected by stainless steel rods. The stopper also had a hole that served as a water vapor outlet. The output of the oscillator was connected to frequency counter 2 by a coaxial cable. The oscillator was fed from a voltage source 3 through the same cable. The measurement results were displayed on the screen of computer 4 .

For the self-oscillator method, the relationship between the frequency of the self-oscillator and the dielectric constant of the material between electrodes of the sensor is given by the formula

$f=\frac{10^{3}}{2 \pi \sqrt{L\left(\varepsilon C_{W}+C_{P}\right)}}$

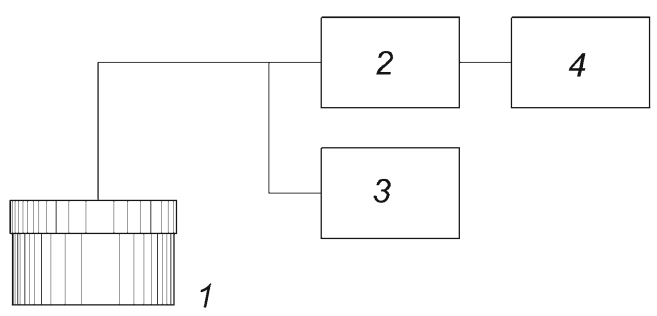

Fig. 1. A block diagram of the measurement setup 
where $f$ is the frequency of the self-oscillator in megahertz, $L$ is the inductance of the self-oscillator circuit in microhenry, $C_{W}$ is the operating capacitance (i.e., the capacitance between the electrodes of the sensor), and $C_{P}$ is the parasitic capacitance (the capacitance of connecting rods and the input capacitance of the self-oscillator transistor) of the sensor in picofarads.

According to the theory of dielectric permittivity of binary systems (24), the dielectric permittivity of a mixture can be expressed in terms of the dielectric permittivities of the components. For the dielectric constant of a frozen biological product in the linear approximation, one can apply the Silberstein-Newton formula:

$\varepsilon=\varepsilon_{1}(1-P)+\varepsilon_{2} P$

where $\varepsilon, \varepsilon_{1}$, and $\varepsilon_{2}$ are the permittivity of the frozen biological material, ice, and water, respectively, and $P$ is the content of the liquid fraction in the frozen material, i.e., the ratio of the liquid phase volume to the volume of the biological material in a vial. The dielectric permittivity of the solid phase (ice) was assumed to be equal to 3.17 (при $-40^{\circ} \mathrm{C}$ ) and that of the liquid phase (water) was 81 . Note that some researchers assume that the dielectric constant of water is lower for low values of $P$ (25). Note that the salts contained in the frozen biological material have little effect on the dielectric constants both of ice and of the liquid phase of the material.

The content of the unfrozen liquid phase in the frozen biologics under study was additionally monitored by NMR spectrometer PAT 20 (Variana).

Comparative study was carried out of relative sensitivities of capacitive and resistivity sensors to liquid phase at various time steps of the sublimation stage. For these measurements, the material to be dried was heated at the beginning, middle, and end of the sublimation stage, and the resistivity of the material and the frequency of the capacitive sensor were measured. The heating was performed after 1, 11.5, and at $20.3 \mathrm{~h}$ of sublimation, respectively.

The freeze drying of a test biological material under the control of a capacitive sensor was carried out with the following control algorithm. Before starting the drying process, the resistivity of a frozen material was set equal to $20 \mathrm{M} \Omega$ by adjusting the temperature and the frequency of the capacitive sensor determined. During the sublimation stage, the frequency of the capacitive sensor was maintained constant by adjusting the temperature of the material to be dried.

In order to take into account the effect of temperature on the parameters $L, C_{W}$, and $C_{P}$ in Eq. (1), we carried out additional experiments and found that the temperature coefficient of frequency (TCF) of the oscillator is equal to $-2.4 \mathrm{kHz} /{ }^{\circ} \mathrm{C}$.

\section{RESULTS AND DISCUSSION}

\section{Freezing Stage}

Figure 2 shows the frequency of the capacitive sensor (curves 1 and 2) and a signal from the resistivity sensor (curves $1^{\prime}$ and $2^{\prime}$ ) during thawing as functions of temperature. Curves 1 and $1^{\prime}$ are obtained for sensors filled with skimmed milk, and curves 2 and $2^{\prime}$ for sensors filled with a protective medium

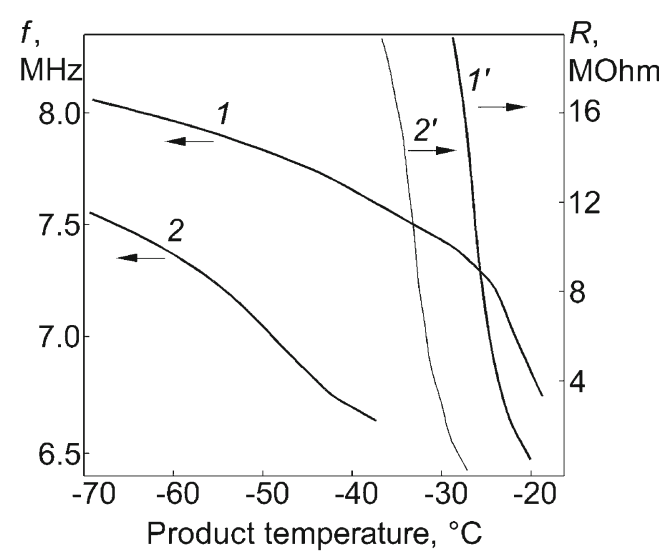

Fig. 2. Frequency of capacitive sensor and signal of resistivity sensor as a function of temperature during thawing

based on peptone. One can see that, as the temperature decreases, the resistivity of the frozen material sharply increases to values that are difficult to measure. This limits the use of the resistivity sensor for controlling the freeze drying process. At the same time, the frequency of capacitive sensor can easily be measured up to a temperature of about $-70^{\circ} \mathrm{C}$. This allows one to control the process of sublimation in the temperature range from -20 to $-70^{\circ} \mathrm{C}$.

Figure 3 shows the fraction of the liquid phase in frozen milk (curve 1) and in frozen protective medium based on peptone (curve 2), calculated from curves 1 and 2 in Fig. 2. Points near curve 1 show the liquid phase fraction in frozen skimmed milk measured during thawing by the NMR method. The dispersion of the liquid phase content in these cases does not exceed $6 \%$. Thus, the permittivity of frozen biological product can objectively describe the liquid phase content.

\section{Sublimation Stage}

Our experiments showed that the horizontal arrangement of electrodes of the capacitive sensor provides practically identical drying conditions for the biological material in the vials and in the capacitive sensor. We believe that this is due to the fact that the arrangement of electrodes did not distort the sublimation front during the whole sublimation stage.

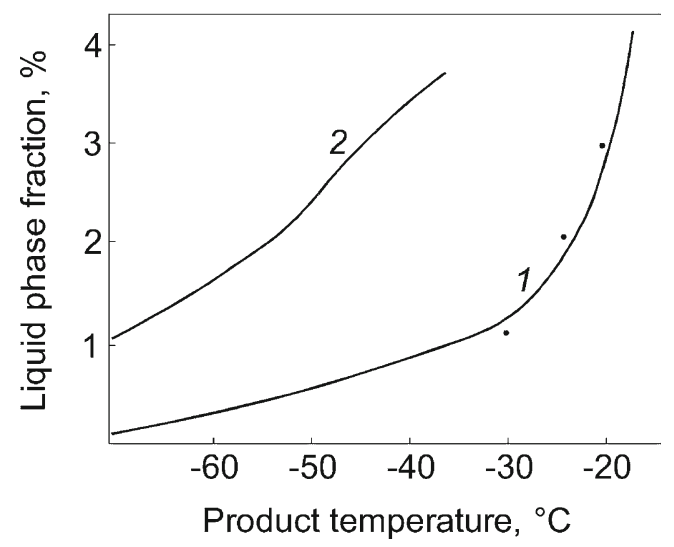

Fig. 3. Percentage of the liquid phase fraction as a function of temperature: 1 -in frozen milk and 2-in frozen protective medium on the base of peptone 

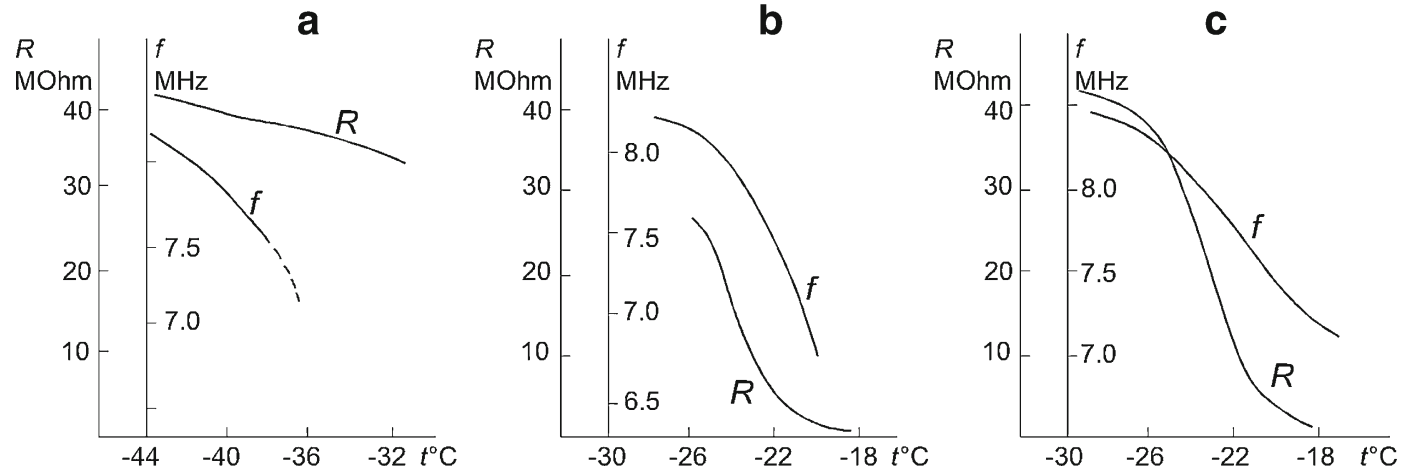

Fig. 4. Comparative sensitivity of capacitive and resistivity sensors to the liquid phase in the frozen biologic at various time steps of the sublimation stage (explanation in the text). a Signals from the capacitive and resistivity sensors versus temperature under heating after $1 \mathrm{~h}$ of sublimation. b, $\mathbf{c}$ Signals from the capacitive and resistivity sensors versus temperature under heating at 11.5 and at $20.3 \mathrm{~h}$ of sublimation

Note also that the measurement of the dielectric permittivity of a biological product adjacent to the bottom of the sensor gives the opportunity to control the drying process during the entire sublimation stage and determines the end of sublimation, because when the sublimation of residual ice between the electrodes of the sensor is completed, the capacitance abruptly decreases and the frequency of the selfoscillator increases. It should also be noted that in our case the heating of the material in the vials is carried out through their bottoms; therefore, the part of the biological product located at the bottom of the vial is most susceptible to foaming. If the amount of liquid phase in a biological product at the bottom of a vial was maintained during the sublimation stage at a level that does not cause foaming, then it would be possible to considerably reduce the drying time and to significantly increase the quality of the end product.

Figure 4 demonstrates the sensitivities of the capacitive and resistivity sensors at the beginning, middle, and end of the sublimation stage. Figure 4a shows the signals from the capacitive and resistivity sensors versus temperature under heating after $1 \mathrm{~h}$ of sublimation. One can see that the frequency of the capacitive sensor decreases with increasing temperature, and then a failure of self-oscillation occurred. The signal from the resistivity sensor in this temperature range varies only slightly. Figure $4 \mathrm{~b}$, c shows signals from the capacitive and resistivity sensors versus temperature under heating at 11.5 and at $20.3 \mathrm{~h}$ of sublimation, respectively. Here, we clearly see that both the frequency of the capacitive sensor and the resistivity of material decrease with increasing temperature and that the decrease in resistivity with heating at the end of the sublimation stage is faster than that in the case when heating is performed at the beginning of the sublimation stage. These results show that the sensitivities of the capacitive and resistivity sensors are dissimilar at different stages of sublimation. At the early stage of sublimation, the capacitive sensor is more sensitive to the content of liquid phase in the biological material, while, in the middle and the late phases of the sublimation stage, the resistivity sensor is more sensitive. This behavior of the sensors can be explained by the fact that, in the middle and the final phases of the sublimation stage, the sensors contain in addition to the frozen biological material and liquid phase a biological material in which the sublimation of ice is completed. The liquid phase can dissolve this material, and its conductivity increases, resulting in increased sensitivity of the resistivity sensor. In contrast, the capacitive sensor responds only to the amount of liquid phase in the material, and the sensor frequency does not depend on its conductivity. Thus, the use of the resistivity sensor at the initial step of the sublimation stage can cause a foaming of the product to be dried. On the other hand, at the end of the sublimation stage, too high sensitivity of the resistivity sensor leads to ungrounded prolongation of this stage.

Figure 5 shows the temperature $t$ of the biological material, the frequency $f$ of the capacitive sensor, and the resistivity $R$ as a function of time $T$ from the beginning of the sublimation stage by the mode when the frequency of the capacitive sensor at the sublimation stage was kept constant. During the drying process, the resistivity of the material was reduced from $20 \mathrm{M} \Omega$ at the beginning of drying to $0.8 \mathrm{M} \Omega$ at the late phase of the sublimation stage. The frequency $f$ of the capacitive sensor started to rise sharply after $10 \mathrm{~h}$ of drying, which indicated that the sublimation front has gone below the upper electrode of the capacitive sensor. After $14 \mathrm{~h}$ of drying, the sharp increase in the frequency stopped. This indicates the end of the sublimation stage and the start of the final stage of drying. Note that the sublimation stage usually lasts $27 \mathrm{~h}$ in the conventional drying mode. We tested the change in the vaccine activity in the proposed and conventional drying modes. It turns out that the difference between the changes in the

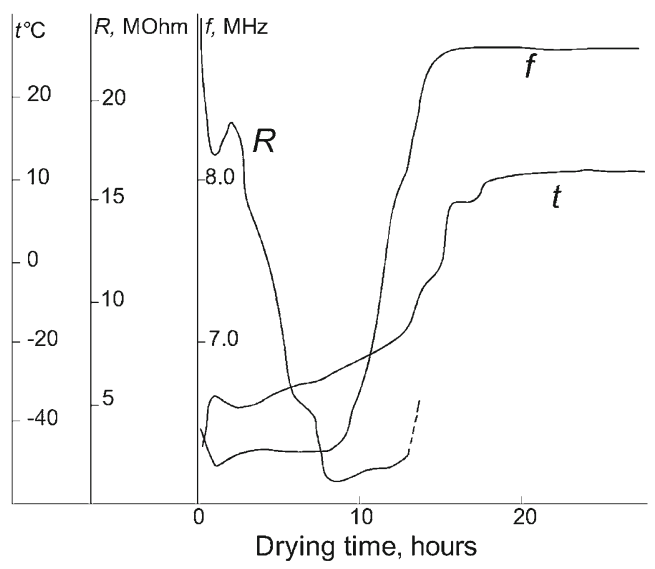

Fig. 5. Temperature of a dryable biological, frequency of capacitive sensor, and specific resistance $R$ of the resistivity sensor as a function of time from the beginning of the drying 
activity of the vaccine dried by the two methods is not statistically significant. Thus, the method proposed for controlling the freeze drying process allows one to expand the control range at the sublimation stage of drying up to $-70^{\circ} \mathrm{C}$ and to reduce the sublimation stage approximately twice without deteriorating the quality of the dried product. Note that we have not observed any change in the shape of the tablets to be dried. Thus, one can conclude that the material's temperature at the sublimation stage does not exceed the collapse temperature $(26,27)$.

\section{Final Stage of Drying}

The final stage of freeze drying for a test biological material was investigated when the primary stage was completed in the conventional mode. Figure 6 shows the frequency dependencies of the capacitive sensor (curve 1) and the temperature of the material in it (curve 2) after $25 \mathrm{~h}$ of drying. Curve 1 has a maximum after $30 \mathrm{~h}$ of drying and then starts to decrease. We have assumed that this decrease of frequency was due to the influence of temperature on the parameters $(L$, $C_{W}$, and $C_{P}$ ) of oscillating circuit. However, the temperature of the dried biological material is practically unchanged after $40 \mathrm{~h}$ of drying, while the frequency of the oscillator continues to decrease. The results shown in curve 1 were recalculated with regard to the TCF, and curve 3 in Fig. 6 shows the corrected dependence of the frequency of the capacitive sensor on temperature. This curve reaches a maximum after $41 \mathrm{~h}$ of drying. Note that the residual moisture of the vaccine at the end of drying, which is determined by the maximum signal of the capacitive sensor, was $1.4 \%$, which was within the optimum residual moisture for this vaccine (1-3\%) (28). This dependence of the frequency on drying time was observed for a sufficiently long sublimation stage. If the sublimation time was reduced to $10-12 \mathrm{~h}$ or less, the time dependence of frequency had no maximum and the frequency grew monotonically, approaching to a maximum value.

It follows from these results that the dielcometry method cannot be used for the quantitative monitoring of the current moisture of biological products at the stage of final drying because the dependence of the capacitive sensor signal on

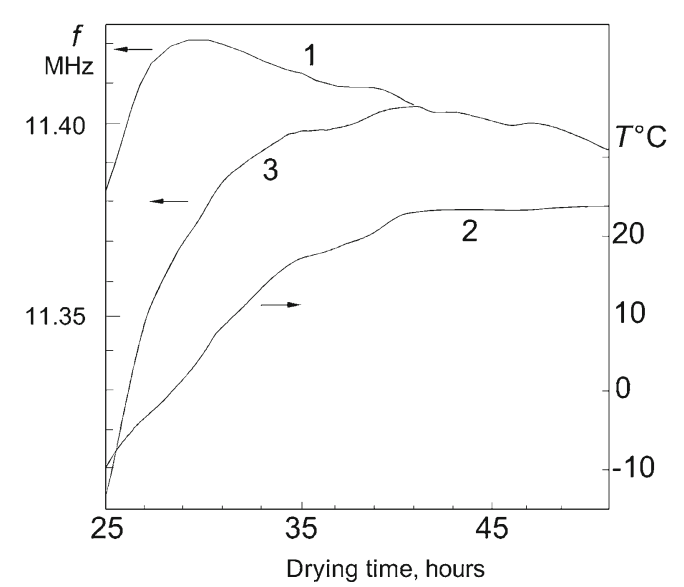

Fig. 6. Dependences on time at the final stage of drying: the frequency of capacitive sensor -1 ; the temperature of biologic in it-2; and curve 1 corrected with the TCF of oscillator-3 the drying time and, hence, the dependence of the biologic moisture on time are ambiguous; moreover, the form of this curve depends on the drying conditions of a biological product at the stage of sublimation. However, we believe that the dielcometry method can be used to determine the optimum residual moisture of biological products directly during the process of drying.

To justify this claim, we first note that, as it was mentioned in the introduction, the dielectric permittivity characterizes the mobility of the charged parts of biological macromolecules: an increase in the dielectric permittivity indicates an increase in the intramolecular motion, while a decrease indicates the slowing down of this motion (29). Note also that freeze-dried biological products are in fact dispersed capillary-porous bodies consisting mainly of biological macromolecules interconnected with each other and water molecules by different chemical bonds. In this case, the increase of the oscillator frequency at the initial stage of final drying can be explained by the fact that, at this stage of drying, weakly coupled water molecules are removed. These molecules have large dipole moment and make a significant contribution to the total dielectric constant of a biological product. Furthermore, water molecules can act as a lubricant for biological macromolecules, thus increasing their mobility. A further removal of moisture from a biological product leads to the complete removal of free water molecules from it, while the remaining water molecules are incorporated into the framework of biological macromolecules and provide their stability. In this case, the dielectric constant of the biological product is minimal. A further removal of water molecules results in the destruction of the structure of biological macromolecules, thus increasing their mobility and the dielectric constant of the material. It can be assumed that the weakening of the frame of biological macromolecules increases their degradation rate during storage. The presence of a minimum of molecular mobility for a certain moisture content of biological products is consistent with the results obtained by other authors. For example, in (30), the mobility of protons in the model biological macromolecules-water system was investigated by the spin-echo NMR method, and it was shown that, at certain moisture, the proton mobility attains its minimum.

In our experiments, the residual moisture of the test biological material fell into the interval of optimal residual moisture when the sublimation stage was rather long and the final stage ended near the maximum frequency of the capacitive sensor. We believe that this maximum corresponds to the minimum of the intramolecular mobility of biological macromolecules. In this case, water molecules existing in a biological material stabilize the skeleton of macromolecules. Further removal of moisture from a biological material destroys this skeleton, and the mobility of macromolecules increases, thus leading to an increase in the dielectric permittivity of the material and a decrease in the frequency of the capacitive sensor. We also believe that a minimum of the intramolecular mobility of biological macromolecules is attained at the optimal residual moisture of biological, when their activity is preserved for the longest time. Thus, we can conclude that the use of a capacitive sensor for monitoring the final stage of freeze drying allows one to complete this stage at the optimal residual moisture of the biological material and improve its quality. 


\section{CONCLUSIONS}

We have proposed a new method for monitoring the freeze drying process of biological materials in vials placed on the shelves of the freeze drier. The state of a biological material is monitored by means of a capacitive sensor mounted on the tray together with vials.

At the stages of freezing and primary drying, the sensor allowed to monitor the fraction of liquid phase in the frozen material up to $-70^{\circ} \mathrm{C}$. Sensor allowed carrying out primary drying by an optimal way without the trial runs. It was shown experimentally that the time of primary drying can be reduced up to two times without loss of product quality. The sensor detects the end of the primary drying stage and the beginning of the secondary drying stage. At the secondary stage, the frequency of the capacitive sensor passed through a maximum which, in our opinion, corresponded to the minimum of the intramolecular mobility of biological macromolecules. We believe also that the minimum of the intramolecular mobility of biological macromolecules is attained at the optimal residual moisture of the material, at which the activity of the material is preserved for the longest time.

\section{REFERENCES}

1. Hsu CC, Ward CA, Pearlman R, Nguyen HM, Yeung DA, Curley JG. Determining the optimum residual moisture in lyophilized protein pharmaceuticals. Dev Biol Stand. 1992;74:255-70.

2. Pikal MJ, Dellerman K, Roy ML. Formulation and stability of freeze-dried proteins: effects of moisture and oxygen on the stability of freeze-dried formulations of human growth hormone. Dev Biol Stand. 1992;74:21-37.

3. Yoshioka S, Aso Y. Correlations between molecular mobility and chemical stability during storage of amorphous pharmaceuticals. J Pharm Sci. 2007;96:960-81.

4. De Beer TRM, Wiggenhorn M, Hawe A, Kasper JC, Almeida A, Quinten T, et al. Optimization of a pharmaceutical freeze-dried product and its process using an experimental design approach and innovative process analyzers. Talanta. 2011;83:1623-33.

5. Tarek M, Tobias DJ. The dynamics of protein hydration water: a quantitative comparison of molecular dynamics simulations and neutron-scattering experiments. Biophysical J. 2000;79:3244-57.

6. Halle B, Denisov VP. Water and monovalent ions in the minor groove of B-DNA oligonucleotides as seen by NMR. Biopolymers. 1998;48:210-33.

7. Pal SK, Zewail AH. Dynamics of water in biological recognition. Chem Rev. 2004;104:2099-123.

8. Sane SU, Wong R, Hsu CC. Raman spectroscopic characterization of drying-induced structural changes in a therapeutic antibody: correlating structural changes with long-term stability. J Pharm Sci. 2004;93:1005-18.

9. Chang L, Shepherd D, Sun J, Ouellette D, Grant KL, Tang XC, et al. Mechanism of protein stabilization by sugars during freezedrying and storage: native structure preservation, specific interaction, and/or immobilization in a glassy matrix? J Pharm Sci. 2005;94:1427-44.
10. Wang B, Tchessalov S, Warne NW, Pikal MJ. Impact of sucrose level on storage stability of proteins in freeze-dried solids: I. Correlation of protein-sugar interaction with native structure preservation. J Pharm Sci. 2009;98:3131-44.

11. Nail SL, Gatlin LA. Advances in control of production freezedryers. PDA J Pharm Sci Technol. 1985;39:16-27.

12. Roy ML, Pika MJ. Process control in freeze drying: determination of the end point of sublimation drying by an electronic moisture sensor. PDA J Pharm Sci Technol. 1989;43:60-6.

13. Louis R. Improvements in and relating to freeze drying and similar processes. Patent BDR. 1964;1:178-787.

14. Genin N, Rene F, Corrieu G. A method for on-line determination of residual water content and sublimation end-point during freeze-drying. Chem Eng Process. 1996;35:255-63.

15. Brulls M, Folestad S, Sparen A, Rasmuson A. In-situ near-infrared spectroscopy monitoring of the lyophilization process. Pharm Res. 2003;20(3):494-9.

16. De Beer T, Vercruysse P, Burggraeve A, Quinten T, Ouyang J, Zhang $\mathrm{X}$, et al. In-line and real-time process monitoring of a freeze drying process using Raman and NIR spectroscopy as complementary process analytical technology (PAT) tools. J Pharm Sci. 2009;98:3430-46.

17. Nail SL, Jiang S, Chongprasert S, Knopp SA. Fundamentals of freeze-drying. In Development and manufacture of protein pharmaceuticals. Springer US, 2002 p. 281-360.

18. Smith G, Duffy AP, Shen J, Olliff CJ. Dielectric relaxation spectroscopy and some applications in the pharmaceutical sciences. J Pharm Sci. 1995;84:1029-44.

19. Rodrigues AC, Viciosa MT, Danede F, Affouard F, Correia NT. Molecular mobility of amorphous s-flurbiprofen: a dielectric relaxation spectroscopy approach. Molecular Pharmaceutics. 2014;11:112-30.

20. Suherman PM, Taylor PM, Smith G. Development of a remote electrode system for monitoring the water content of materials inside a glass vial. Pharm Res. 2002;19:337-44.

21. Smith G, Polygalov, Arshad MS, Page T, Taylor J, Ermolina I. An impedance-based process analytical technology for monitoring the lyophilisation process. Int J of Pharm. 2013;449:72-83.

22. Smith G, Arshad MS, Polygalov E, Ermolina I. Factors affecting the use of impedance spectroscopy in the characterization of the freezing stage of the lyophilisation process: the impact of liquid fill height in relation to electrode geometry. APPS Pharm Sci Tech. 2014;15:261-9.

23. Sokoll T, Jacob AF. Self-oscillating measurement system for in situ-monitoring moisture in buildings. Instrum Meas, IEEE Transac. 2014;63:2911-8.

24. Brown WF. Dielectrics. Berlin: Springer; 1956.

25. Oehme F. Dielektrische Messmethoden zur quantitativen Analyse und für chemische Strukturbestimmungen. Verlag Chemie, 1962: Vol. 70.

26. Pikal MJ, Shah S. The collapse temperature in freeze drying: dependence on measurement methodology and rate of water removal from the glassy phase. Int J Pharm. 1990;62:165-86.

27. Greco K, Mujat M, Galbally KL, Hammer DX, Ferguson RD, Iftimia N, et al. Accurate prediction of collapse temperature using optical coherence tomography based freeze-drying mi microscopy. J Pharm Sci. 2013;102:1773-85.

28. May JC, Wheeler RM, Etz N, Del Grosso A. Measurement of final container residual moisture in freeze-dried biological products. Dev Biol Stand. 1992;74:153-64.

29. David SP, Smith G. Dielectric analysis as a tool for investigating the lyophilization of proteins. PSTT. 1998;1:108-17.

30. Aksenov SI. The state of water and its role in the dynamics of biological structures. Doctoral (Phys. - Math.) dissertation. Moscow State University; 1979. 